\section{DISPOSITIFS DE SECURITÉ}

\author{
APPLIQUUS AUX
}

\section{Barrages de la Bouillouse et de Marklissa}

BARRAGE DE LA BOUILlOUSE

Le barrage-réservoir de la Bouillouse, actuellement en construction, avait été depuis longtemps projeté par le Ministère de l'Agriculture en vue de la régularisation du débit de la rivière la Tet. Cette rivière, en effet, n'est, à proprement parler, qu'un torrent alimenté seulement d'une façon intermittente, et souvent très brusque, par les fortes pluies d'automne ou par la fonte des neiges et qui, pendant les mois de sécheresse, ne laisse couler qu'une faible quantité d'eau, insuffisante d'ailleurs pour desservir les nombreux canaux d'irrigation qui sillonnent la fertile plaine du Roussillon.

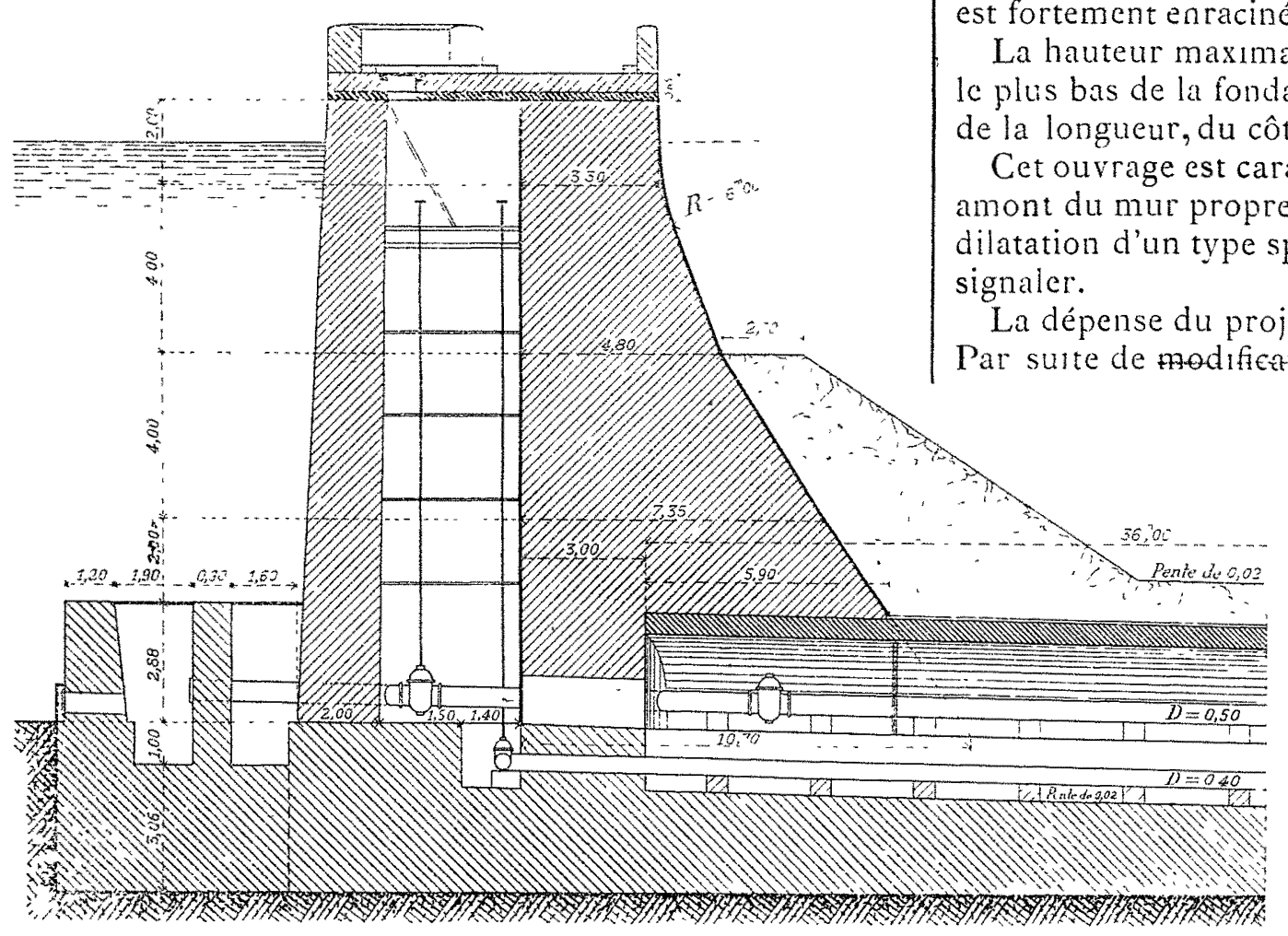

giques faites dans le voisinage et les considérations climatologiques ont établi qu'il serait possible d'emmagasiner annuellement treize millions de mètres cubes d'eau. La surface moyenne du réservoir étant de roo hectares, on a donc été amené à fixer la hauteur de la retenue normale à I 3 mètres.

Le barrage, une fois terminé, aura une longueur totale de $363 \mathrm{~m}$., en ligne droite, sauf une légère inflexion suivant une courbe de $\mathrm{I} 7 \mathrm{~m}$. de rayon, vers l'extrémité de rive gauche, pour dégager l'entrée du canal de fuite du déversoir. Il est enzastré, partout, d'un mètre au moins dans le granit compact. Le rocher est à nu sur toute la rive gauche de la vallée; sur l'extrémité rive droite, il s'abaisse jusqu'à une profondeur de $15 \mathrm{~m}$. au-dessous du sol. Le terrain, audessus du rocher, est constitué par un dépôt glaciaire, com. posé de terre argileuse provenant de la décomposition des schistes et des granits, et contient des cailloux et des blocs roulés de toute dimension. De ce côté de la vallée, le barrage est fortement enraciné dans le coteau:

La hauteur maxima du mur, du couronnement au point le plus bas de la fondation, a lieu sensiblement vers le tiers e la longueur, du côté droit; elle sera de 22 mètres.

Cet ouvrage est caractérisé par l'adoption d'un masque en spécial qu'll nous paraît intéressant de gnaler.

La dépense du projet primitif s'élevait à i 200000 francs. Par sulte de modifiertions prescrites par la décision minis-

FIG. I. - Coupe transversale du barıage de la Bouillouse suivant l'axe de la galerie de prise d'eau.

L'étude de ce projet a été reprise par le Ministère des Travaux Publics pour l'alimentation de l'usine hydroélectrique qui doit fournir l'énergic nécessaire à la traction des trains sur le chemin de fer électrique d'intérêt général, à voie de $1 \mathrm{~m}$., de Villefranche-de-Conflent à Bourg-Madame, ligne déclarée d'utilité publique et concédée à la compagnie des Chemins de fer du Midi par la loi du 4 mars igo3. Cette rouvelle destination permettera en même temps de réaliser l'amélioration projetée au point de vue agricole.

Le barrage est établi en travers du lit de la Tet, à $\mathrm{i} 3 \mathrm{~km}$. environ en amont de Mont-Louis (Pyrénées-Orientales), à l'extrémité aval de la Grande Bouillouse. Ce marais, d'une surface d'environ 100 hectares, sensiblement horizontal, est situé à 2.000 mètres d'altitude, au centre du massif montagneux que dominent les pics du Carlitte $(2.92 \mathrm{I} \mathrm{m}$.), de Puig-Péric (2.81o m.) et de Roque-Rouge (2.834 m.).

Le bassin hydrologique de la Tet, en amont du barrage, a une surface de 2.800 hectares. Les observations météorolo- térielle approbative, de changements des lieux de provenance de certains matériaux, l'évaluation a dủ être portée à I $700000 \mathrm{fr}, \mathrm{y}$ compris une somme à valoir de $220000 \mathrm{fr}$. Le prix de revient du mètre cubc de capacité ressort donc à o fr. 13. C'est l'un des plus bas qui ait été réalisé (I).

Bien qu'à moitié construit, ce barrage a déjà rendu de grands services aux riverains de la Tet, en assurant la marche régulière des usines hydrauliques d'aval, et en alimentant d'eau pendant l'été, pour la première fois, les canaux d'irrigation de la plaine du Roussillon. De plus, pendant les inondations qui ont dévasté dernièrement le Midi, ce réservoir a considérablement amorti la crue de la Tet (2).

(I) Notons toutefois qu'au barrage de l'Urft, qui crée un réserrolr de 45,5 millions de $\mathrm{m}^{3}$ ayec une hauteur de $58 \mathrm{~m}$., la dépense n'a éte que de ofr. io par mètre cube. Pour la description de ce barrage, se reporter au no de juin roo6 de La Houlle Blanche

(2) Voir La Houille Blanche de janvier $1908, \mathrm{p} .3$. 
seur, portant en son milieu une ondulation longitudinale; recouvrira le joint à l'amont, sur toute sa hauteur. Cette lame, dont les extrémités latérales sont retournées à angle droit, sera scellée au plomb, au moyen de rainures pratiquées dans les moellons, de chaque côté du joint ; elle sera, en outre, invariablement fixée au moyen de boulons galvanisés scellés dans la maçonnerie.

Lorsque le masque se dilatera, la courbure de l'ondulation s'accentuera; elle s'applatira lors de la contraction

Prises d'call. - Au droit du puits central, il est ménagé à travers le barrage, et sur $30 \mathrm{~m}$. au-delà; une galerie voûtée, de $3 \mathrm{~m}$. d'ouverture et de $2^{\mathrm{m}} 75$ de hauteur sous clef, destinée à recevoir les tuyaux d'écoulement et à servir de chambre de manœurre.

Les prises d'eau sont au nombre de deux, l'une destinée à l'alimentation normale, et l'autre à la vidange du réservoir; toutefois, cette dernière prise pourra au besoin servir à l'alimentation. Elles sont constituées chacune par un tuyau en fonte de $0^{\mathrm{m}} 5 \mathrm{o}$ de diamètre.

Ces deux tuyaux ont chacun leur orifice amont dans un puisard spécial, établi dans le réservoir. Ils traversent le puits central et aboutissent dans la galerie, où ils sont posćs sur des socles en maçonnerie de om 40 de hauteur, et déboulchent à l'aval dans une sorte de puisard, à $1 \mathrm{~m}$. environ en contrebas du niveau d'écoulement du canal de prise d'eau. Cette dernière disposition a pour but d'éviter la congélation de l'eau à la sortie des tuyaux.

Chaque tuyau sera muni de deux robinets-vannes, modèle de la ville de Paris; l'ùn placé à l'extrémité amont de la galerie, et l'autre, dit de secours, dans le puits central oú il sera manœuvré, au moyen de tiges, du sommet du barrage.

Le tuyau de drainage sera également muni de robinetsvannes, pour éviter l'écoulement de l'eau en cas d'accident à l'un des puits du masque.

La galerie sera fermée par une triple porte vitrée, de façon à éviter toute introduction d'air froid.

Déversoir. - A l'extrémité du barrage, sur la rive gauche, il sera établi un déversoir en maçonnerie, arasé à la cote 2013, de manière à permettre le déversement des eaux surabondantes en temps de crues. Ce déversoir aura $40 \mathrm{~m}$. de longueur, et sera disposé en plan suivant un arc de cercle de $130 \mathrm{~m}$. de rayon s'appuyant perpendiculairement sur le mur de barrage. Les eaux de trop-plein se déverseront dans un canal de fuite, susceptible de débiter $130 \mathrm{~m}^{3}$ à la seconde avec une lame d'eau de $\mathrm{I}$ 50 . Ce canal débouchera dans la Tet à $130 \mathrm{~m}$. environ en aval du barrage.

\section{BARRAGE DE MARKLISSA}

Ce barrage a été construit de Igor à 1905, près de la - ville de Marklissa (Silésie), sur le Queis, affluent du Bober, qui se jette lui même dans l'Oder. Ce cours d'eau est sujet à des crues subites qui peuvent faire brusquement varier son débit de $1 \mathrm{~m}^{3}$ à l'étiage jusqu'à plusieurs centaines de mètres cubes; et c'est à la suite des dégâts considérables qui ont été occasionnés par une crue de 1897 que sa création a été décidée.

Le barrage a $45 \mathrm{~m}$. de hauteur au-dessus du ropher de fondation, mais ce n'est que la partie supérieure du réservoir ainsi obtenu, sur une trinche de $9 \mathrm{~m}$. 80 , qui est utilisée pour l'emmagasinement et l'évacuation des crues, en fournissart le volume de to millions de mètres cubes reconnu nécessaire à cet effet. La partie inférieure est destinée à alimenter les turbines d'une usine hydro-électrique située à l'aval du barrage; son volume est de 5 millions de $\mathrm{m}^{3}$ et constitue une réserve capable d'augmenter dans une certaine mesure le débit du Queis pendant la saison sèche. La hauteur normale de la chute sous laquelle travaillent les turbines est de $30 \mathrm{~m}$., et si l'on vient à vider la réserve jusquà ce qu'elle soit réduite à I million de $\mathrm{m}^{3}$, cette hauteur sera encore de 20 mètres.

La création du réservoir a coûté 32 Io 000 marks, soil 4 millions de francs. Le cube d'emmagasinemeut des crues étant de 10 millions de $\mathrm{m}^{3}$, le prix de revient du mètre cube de crue absorbée revient donc o fr. 40 ; si l'on tient compte des 5 millions de $\mathrm{m}^{3}$ supplémentaires qui peuvent être utilisés comme réserve par l'usine hydro-électrique, le prix de revient s'abaisse à o fr. 266, qui est bas par lui-mềme; mais qui, néanmoins, est le double plus élevé que celui obtenu au barrage de la Bouillouse où les conditions géogra. phiques sont plus favorables.

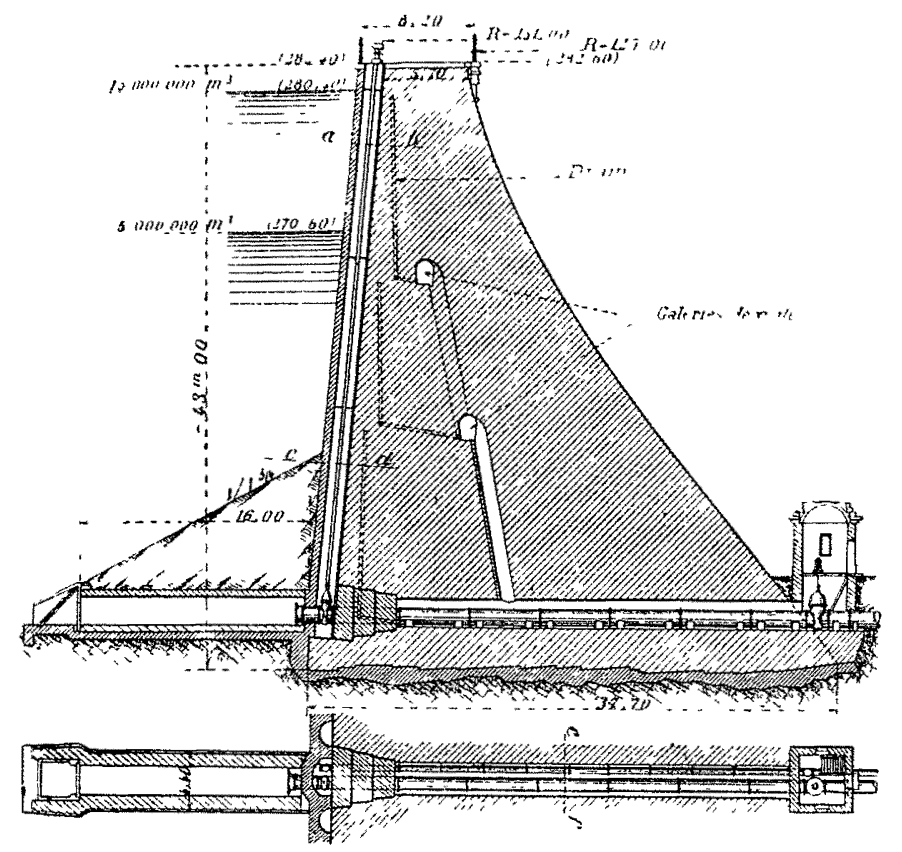

FIG 3. - Coupe verticale et horizontale du barrage de Marklıss suivant une galerie de prise d'eau (r).

L'usine hydro-électrique est établie pour pouvoir développer 2800 chevaux pendant 12 heures par jour. Elle comporte 5 turbines de $700 \mathrm{HP}$ actionnant des alternateurs; ceux-ci fournissent du courant à Io ooo volts qui distribué sous cette tension dans un rayon de 50 kilomètres.

La dépense prévue, tant pour l'usine génératrice que pour l'achèvement du réseau primaire de distribution à haute tension, s'élève à I 500000 marks ( 900000 fr.) et l'on compte vendre en gros l'énergie à raison de i 20 à I 40 markşs ( 150 à $170 \mathrm{fr}$.) le cheval-an. En admettant que les dépenses d'exploitation et d'entretien s'élèvent à 20 pour 100 environ de la recette, le bénéfice net annuel s'élèverait à un peu plus $360000 \mathrm{fr}$., ce qui, pour une dépense de premier établissement de 6 millions en nombre rond, ferait un revenu annuel d'un peu plus de 6 pour 100 , chiffre fort rémunérateur, si l'on tient compte du but humanitaire qui a présidé à la construction de cet ouvrage.

Le profil de ce barrage, qui cst représenté par la figure 3 ci-jointe, a été déterminé par le professeur InTze d'Aix-laChapelle, et est analogue aux autres barrages allemands

(1) Cette figure, ainsi que les suivantes, sont extraites d'un article que M. Dumas a consacré à ce barrage dans un récent numéro du Génie Civil. 
établis par cet ingénieur (I). Le mur est établi en plan suivant une forme courbe très prononcée. La vallée, au point où il est implanté, n'a que $30 \mathrm{~m}$. de largeur à la base et i ro m. au sommet.

La caractéristique principale de ce barrage réside dans le surcroît de précautions qui ont été prises pour assurer sa conservation et pour rassurer les riverains d'aval, auxquels la construction du barrage causait de vives appréhensions, et dont beaucoup déclaraient même préférer les risques de nouvelles inondations à ceux provenant de la rupture d'un pareil ouvrage. Ces précautions sont de deux ordres assez différents : ${ }^{\circ}$ Dispositions employées contre les sous pressions susceptibles d'être développées sur les joints horizontaux; $2^{\circ}$ dispositions empêchant le barrage d'être surmonté par les crues.

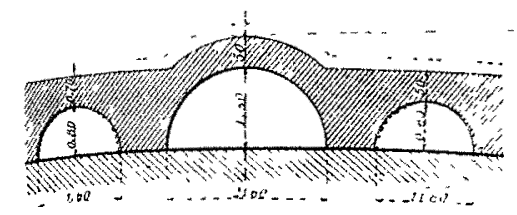

FIG 4 - Coupe suirant $a b$ et $c d$.

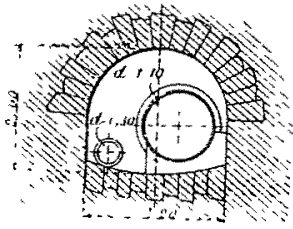

FIG. 5. - Coupe suivant ef.
Contre les sous pressions, on a fait emploi d'un masque établi en avant du parement amont, et, en outre, on a ménagé des drains dans le corps même du barrage, ainsi que le montre la figure 3 . Ces drains aboutissent à deux galeries horizontales de visites, réunies entre elles et aux galeries inférieures de prise d'eau par des puits inclinés, et permettant, d'après le débit des drains, de s'assururer à tout instant de l'état de conservation des maçonneries. S'il est vrai qu'en pareille matière deux précautions valent mieux qu'une, il nous semble cependant qu'on a fait là une dépense inutile, le dispositif des drains étant suffisant, d'autant plus que la pression élastique à l'amont en charge est supérieure à celle de l'eau, et que le barrage est établi en plan suivant une courbe très accentuée, dans une vallée assez étroite, ce qui augmente considérablement sa résistance (2).

Contre les crues, on a employé des déversoirs avec émissaires souterrains d'un type spécial, concuremment avec les galeries de dérivation.

Les maçonneries du mur ont été faites avec un mortier mixte de ciment portland et de pouzzolanne, composé (en volume) de 5 parties de ciment, 4 de trass, 2,64 de chaux éteinte et 20,4 de sable. A trois mois, ce mortier accusait une résistance de 30 à $40 \mathrm{kgs}$ par $\mathrm{cm}^{2}$ à la traction, et de I 24 kgs à la compression.

Le parement amont du mur a été recouvert d'un enduit de ciment, recouvert lui-même d'une couche d'asphalte, contre laquelle vient s'appuyer le mur de masque qui est représenté en coupe par la figure 4 .

(I) Voir dans La Houille Blanche de juin 1907 , le chapitre que nous avons consacré aux barrages allemands, ainsi que les critiques que nous avons formulées contre l'adjonction d'un remblais à la base amont du barrage.

(2) Dans le tırage à part de nos précédents articles sur les barrages (Gratier et Rey, à Grenoble, 1907), nous avons démontré, p. I6r, que si un barrage en voùte était établi avec un profil tel que la pression élastique à l'amont en charge soit égale à celle de l'eau, dans l'hypothèse ou le barrage ne travaille que par son poids, cette pression elastıque était en réalité bien plus grande, et devenait égale à la pression moyenne.

En supprimant les galeries horızontales, et les remplaçant par un ceux de nombre de puits disposés sensiblement à la même place que ceux de la fig. 3, on retrouverait le dispositif que nous avons indiqué drains atre brochure ( $p$. 82 à 84 ) comme combinaison du système des drains avec celui à évidement central de M. Figarr.
Prise d'eau. - La prise d'eau pour l'alimentation des turbines de l'usine hydro-électrique est faite au moyen de deux conduites en fonte, placées séparément dans une galerie inférieure ménagée de chaque côté de l'ancien lit du Queis. Elles sont prévues pour pouvoir débiter $6 \mathrm{~m}^{3}$ par seconde, et sont commandées chacune par deux vannes; l'une, cylindrique, est placée dans une cabine à l'aval, l'autre, dite de sécurité, est plate et est manœuvrée du sommet du barrage au moyen d'une longue tige. Dans la même galerie, et à côté de chacune des conduites de prise d'eau, on a établi une conduite de plus petit diamètre qui recueille l'eau des drains et du mur de garde.

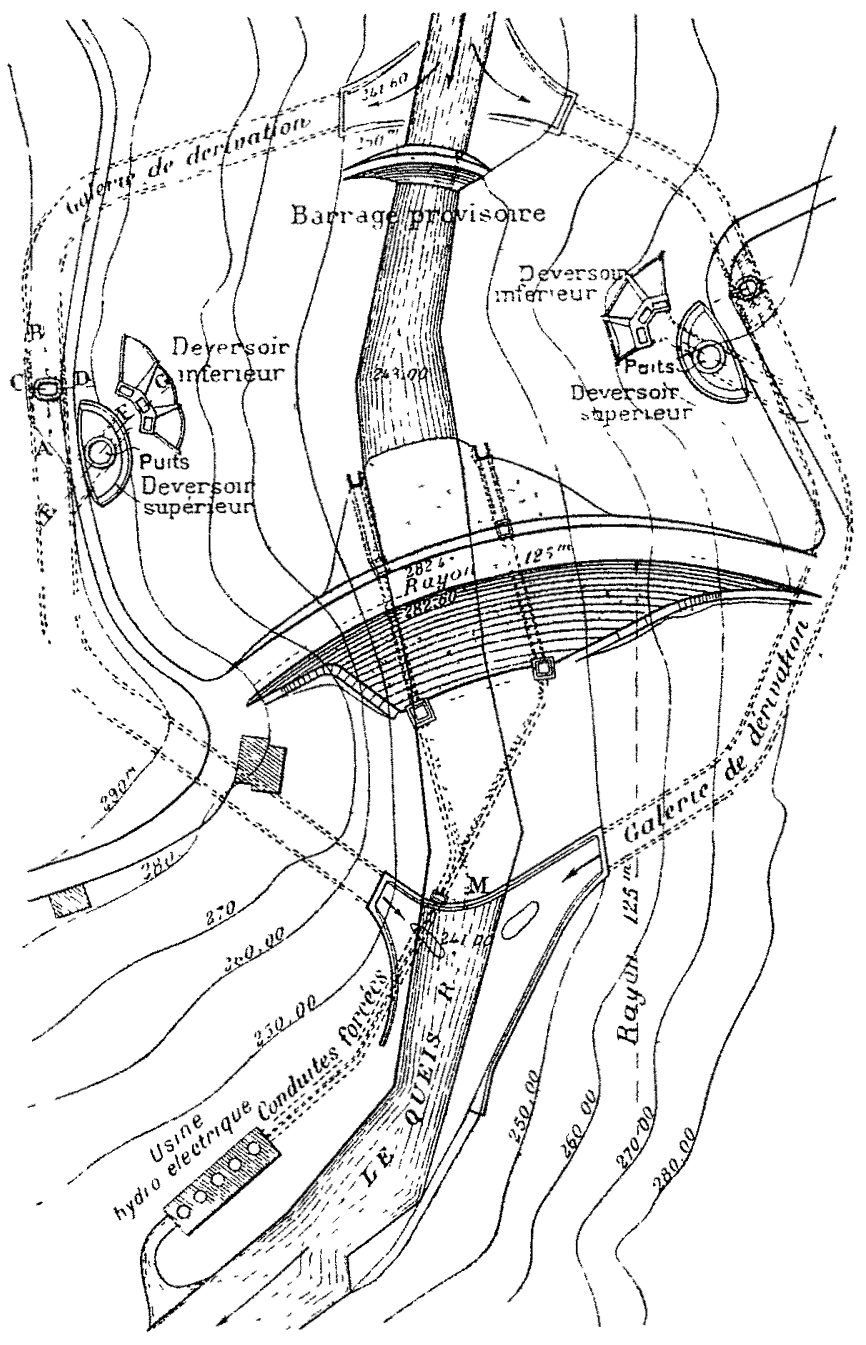

Fig. 6. - Plan d'ensemble du barrage et des ouvrages accessorres.

Galeries de dérivation. - De chaque côté du Queis, on a percé dans les flancs de la vallée une galerie de dérivation, contournant la base de fondation du barrage, de $7 \mathrm{~m}$. de diamètre extérieur, qui a permis de dériver les eaux de la rivière pendant la construction du mur, et qui, depuis l'achèvement de celui-ci, sert à l'évacuation des crues.

Entre les points marqués $A$ et $B$ sur le plan, chaque galerie a été obturée par un massif en maçonnerie formant tampon de $17 \mathrm{~m}$. de longueur, dans lequel on a noyé un faisceau de 3 conduites de $\mathrm{I} \mathrm{m}_{\mathrm{I}}$ o qui, lorsque l'eau est à la cote 270,60 peuvent débiter $55 \mathrm{~m}^{3}$, ce qui, pour l'ensemble des 2 galeries, correspond aux 1 ro $\mathrm{m}^{3}$ qu'il est possible de laisser écouler dans le lit du Queis sans danger pour les riverains.

Ces conduites sont munies de vannes qui sont manœuvrées du niveau supérieur du bassin au moyen de longues tiges loğées dans un puits de visite représenté en C D sur le plan. 
Une petite conduite de o m 10 sert à faire écouler l'eau qui peut s'amasser dans le puits.

Déversoirs. - De chaque côté de la vallée, on a ménagé deux déversoirs établis à des niveaux différents, mais communiquant avec un même puits incliné, de $5 \mathrm{~m}$. de diamètre, qui aboutit à l'une des galeries de dérivation, un peu à l'aval du tampon maçonné qui les obture.

Chacun des déversoirs inférieurs est arrasé à la cote 270,60, et est divisé en 3 parties égales, ainsi que le montre la figure 9 . Il communique avec le puits incliné par l'intermédiaire d'une galerie presque horizontale, de $4 \mathrm{~m}$. de diamètre. Entre cette galerie et chacun des 3 compartiments du déversoir, on a disposé un système de vannage, représenté par la figure 8 , composé d'une partie fixe $c$ et de delix parties mobiles $a$ et $b$.

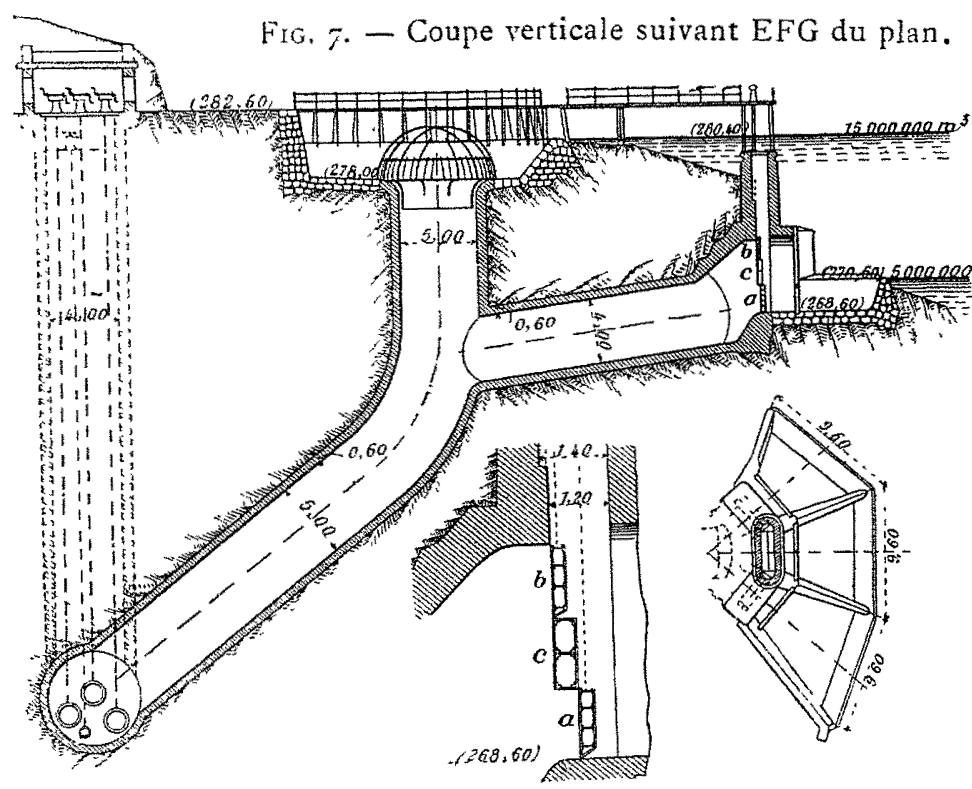

Galerie de dérivation

Fig. 8 et 9. - Coupe verticale des vannes et disposition en plan d'un déversoir interieur

En temps ordinaire, les vannes inférieures $a$ sont ouvertes, et laissent écouler l'eau qui passe par dessus le déversoir. Si l'on veut maintenir le niveau du réservoir au dessus de la normale, on ferme ces vannes $a$, et l'on lève les vannes $b$, de manière à faire écouler. l'eau par dessus $c$ qui forme à son tour déversoir. Cette disposition permettra d'augmenter la capacité utile de la réserve de l'usine hydraulique, et de la porter à 8 millions de $\mathrm{m}^{3}$, lorsque, au bout de quelques années, on connaîtra mieux le régime des oscillations du niveau de l'eau dans le réservoir.

Lorsque le niveau de l'eau s'élève à $\mathrm{I} m$. au-dessus des déversoirs, ceux-ci débitent les I $10 \mathrm{~m}^{3}$ admissibles à l'aval du barrage. On ferme alors les vannes inférieures $a$, et l'on règle le débit des déversoirs au moyen des vannes supérieures $b$, afin de diminuer la pression de ces vannes contre leur guidage et de faciliter leur mancuvre

Chacun des déversoirs supérieurs" est disposé en plan en forme de lentille et est arrasé à la cote 280,40 , soit à $2 \mathrm{~m}$. en contrebas de la crête du barrage, et communique directement avec le puits incliné.

La crue de 1897 (la plus forte constatée), qui a motivé la construetion du barrage, avait atteint $780 \mathrm{~m}^{3}$ à la seconde. Si une pareille crue venait à se produire lorsque le réservoir est complètement plein, chacune des deux galeries de dérivation aukait à évacuer $390 \mathrm{~m}^{3}$. Avec un diamètre de $7 \mathrm{~m}$., la vitesse de l'éau serait de 10 m.à la seconde. Bien que fort imprabable, on a pourtant prévu que ce cas pourrait se produire, ce qui a conduit a revêtir les parois de cette galerie avec des pierres de taille jusqu'aux puits, et avec un garnis. sage en béton à l'amont. Le diamètre intérieur a été de là sorte réduit à $5 \mathrm{~m} 80$, ce qui porte la vitesse à 14 mètres.

Il a été nécessaire de consolider le raccordement de là partie inférieure des puits avec les galeries correspondantes', afin de résister au choc de l'eau arrivant avec une grande vitesse. Pour cela, on a adopté un revêtement métallique, en tôles de 8 à ro mm. d'épaisseur entourées de béton. Il parai. trait que ce blindage n'a pas donné toute satisfaction, et il aurait été préférable de prolonger les puits au-dessous dela génératrice inférieure des galeries, de manière à constituer une fosse créant un matelas d'eaù, et de relier cette fossé avec la galeric de dérivation.

\section{H. Bellet.}

\section{La Déviation da Colorado}

Le Salton Sea (lac Salton) constituait autrefois l'cxtrémité nord du golfe de Californie : mais le Colorado, qui se jetait alors dans ce golfe un peu avant son extrémité, constitua, par ses dépôts, un delta qui, allant toujours en s'élargissant et se surélevant, ferma peu à peu le golfe, et isola le Salton Sea à l'intérieur des terres. Sous l'effet de l'évaporation intense qui se produit dans ces régions, le lac ne tarda pás á voir son niveau baisser bien au-dessous de celui de la mer et sa superficie se réduire considérablement. Jusqu'en 1893, la bande de terre qui sépare le Salton Sea du fond du golfe de Californie n'était qu'un désert aride, coupé par la frontière du Mexique et des Etats-Unis.

En 1893, on imagina de faire une-prise d'eau sur le Colo. rado et de dériver une partio de ses eaux dans un de ses anciens lits, l'Alamo River, presque toujours à sec, de manière à irriguer ce qui constituait jadis l'extrémité sud du Salton Sea et qu'on appelle maintenant l'Impcrial Valley; et l'Alamo River prit lo nom d'Imperial Canal. Cette pre:

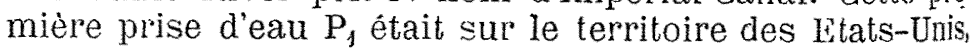
mais comme elle s'était ensablée, on en fit une seconde $\mathrm{P}_{\text {? }}$ à peu de distance de la première, en territoire mexicain.

Les résultats acquis par les irrigations ainsi obtenues furent si excellents que les colons affiuèrent dans l'Imperial Valley. La quantité d'eau dérivée ne tarda pas à devenir iisuffisante, d'autant plus que la nouvelle prise s'ensablait comme la première, malgré des dragages continuels, aussi, devant les réclamations des colons, se hâta-t-on, au début de la saison chaude de 1904, de faire une troisième prise $P_{*}$ en face d'une île, au moyen d'une tranchée de 15 mètres dé large sur $4^{\mathrm{m}} 80$ de profondeur, débouchant immédiatement dans l'Imperial Canal.

Dès la première crue, la prise $\mathrm{P}_{3}$ ne tardà pas à s'élargir, et à livrer passage à une quantité d'eau qui devint de plusen plus considérable, si bien que le Colorado quitta son ancien lit pour se jeter dans le Salton Sea, et il le fit d'autant plus facilement que la pente était plus forte de ce côté que du côté du golfe. Le lac ne tarda pas à déborder et à envahin l'Imperial valley qui fut inondée, ce qui causa d'incalculables dommages. Six tentatives infructueuses furent fáites pour barrer d'abord le canal, puis le nouveau lit du Colorads, quand tout.le fleuve s'y précipita en 1906.

Au printemps 1905, la brèche avait $31 \mathrm{~m}$. de large et $6 \mathrm{~m}$ : de profónd. On battit alors des pieux en travers du cạnal on les moisa, et on constitua en. A un barrage rudimentair? avec des fascinages et des sacs de sable. Mais à la première crue cet ouvrage fut emporté.

En juin 1905 ,on battit à nouveau deùx rantrs dé pieux,enB, 